hep-th 9902010

\title{
A note on a relation between the Killing spinor and Einstein equations
}

\author{
Ali Kaya 田 \\ Center for Theoretical Physics, Texas A\& M University, \\ College Station, Texas 77843, USA.
}

\begin{abstract}
It is shown that, under certain conditions, the existence of a Killing spinor on a bosonic background of a supergravity theory implies that the Einstein equations are also satisfied. As an application of the theorem, we obtain a new black fivebrane solution of $\mathrm{D}=11$ supergravity, which has $K 3 \times R$ topology and preserves $1 / 4$ 'th supersymmetries of the theory.
\end{abstract}

\footnotetext{
${ }^{1}$ partially supported by Scientific and Technical Research Council of Turkey (Tübitak)

${ }^{2}$ also at Department of Physics, Bog̃azici University, İstanbul,Turkey.
} 
In string/M theories, solitons are solutions of the corresponding supergravity equations of motion (for a review see [1], [2]). Generically, they can be interpreted as $p$ dimensional extended black holes (p-branes) and characterized by few constants, like the mass and the charges of the antisymmetric tensor fields. The extreme members of black p-branes, which are obtained when the mass and charge saturate a Bogomolnyi type of bound, are supersymmetric and have stable multi-source generalizations. A background is supersymmetric, if it is invariant under some supersymmetry transformations of the theory, whose parameters are called Killing spinors. The fact that, the states related to a supersymmetric soliton should be in the spectrum at any coupling of the theory, increases the potential importance of such solutions [3].

In many cases, supersymmetric solitons can be obtained by writing suitable ansatzs, which solve the Killing spinor and all but the Einstein field equations from the beginning [1]. Then the Einstein equations are solved to determine unknown functions. This indicates that there may be a connection between the two equation systems. In this paper a theorem is proven which shows that, when the ansatz for the fields and geometry is chosen in a suitable way, the existence of a Killing spinor implies that the Einstein equations are also satisfied. We hope that this observation will be helpful in finding new and interesting supersymmetric solutions.

Let us consider a bosonic background $\left(g_{M N}, F_{M N P Q}\right)$ of $\mathrm{D}=11$ supergravity theory [4] which obeys:

$$
\begin{gathered}
R_{M N}=\frac{1}{3}\left(F_{M}{ }^{P Q R} F_{N P Q R}-\frac{1}{12} g_{M N} F^{P Q R S} F_{P Q R S}\right), \\
\nabla_{Q} F^{Q M N P}=\frac{1}{(24)^{2}} \epsilon^{M N P A_{1} \ldots A_{8}} F_{A_{1} . . A_{4}} F_{A_{5} . . A_{8} .} .
\end{gathered}
$$

The linearized Rarita-Schwinger equations on this background may be written as:

$$
\Gamma^{M N P} D_{N} \psi_{P}=0
$$

where the supercovariant derivative $D_{M}$ is given by

$$
D_{M}=\nabla_{M}+\frac{1}{144}\left(\Gamma_{M}^{P Q R S}{ }_{M}-8 \delta_{M}^{P} \Gamma^{Q R S}\right) F_{P Q R S},
$$

and $\nabla_{M}$ is the usual covariant derivative acting on spinors. Let us further consider a spin $3 / 2$ field $\psi_{M}$, which is obtained by the action of the supercovariant derivative on an arbitrary Majorana spinor $\epsilon$ :

$$
\psi_{M}=D_{M} \epsilon
$$


An interesting question about this spin $3 / 2$ field is whether it solves the linearized Rarita-Schwinger equations (3). The answer is immediately yes, since an affirmative statement is equivalent to the invariance of $\mathrm{D}=11$ supergravity at the linearized fermionic level [5. To verify this let us insert (5) into the field equations (3). After a relatively long but straightforward calculation and using only the equations (2) for the 4-form field, (3) becomes

$$
\left(G_{M N}-T_{M N}\right) \Gamma^{N} \epsilon=0
$$

where $G_{M N}=R_{M N}-\frac{1}{2} g_{M N} R$ is the Einstein tensor and

$$
T_{M N}=\frac{1}{3}\left(F_{M}{ }^{P Q R} F_{N P Q R}-\frac{1}{8} g_{M N} F^{P Q R S} F_{P Q R S}\right)
$$

is the energy momentum tensor. The presence of the Einstein tensor in equation (6) is due to the following identity:

$$
\Gamma_{M}{ }^{N P} \nabla_{[N} \nabla_{P]} \epsilon=\frac{1}{8} \Gamma_{M}{ }^{N P} R_{N P R Q} \Gamma^{R Q} \epsilon=\frac{1}{2} G_{M N} \Gamma^{N} \epsilon .
$$

Not surprisingly the terms, which contain $\nabla_{M} \epsilon$, are canceled. This is because $D_{M}$ is a certain connection defined on the spinor bundle. The curvature $\Omega_{M N}$ of this connection is given by

$$
D_{[M} D_{N]} \epsilon=\Omega_{M N} \epsilon,
$$

which certainly does not have a term containing the derivative of $\epsilon$. Equations (6) are satisfied because $G_{M N}-T_{M N}=0$ are nothing but the Einstein equations (1), which are obeyed by the background. Therefore we ensure, once more, the linearized supersymmetry invariance of $\mathrm{D}=11$ supergravity.

Now, we try to use the results of the above calculations to prove a new theorem. We assume this time that the given bosonic background does not necessarily solve the Einstein equations (1), but it again obeys the 4-form field equations (2). We further assume that there exist a Killing spinor $\epsilon_{0}$,

$$
D_{M} \epsilon_{0}=0
$$

and the Einstein and the energy momentum tensors have only diagonal entries in an orthonormal basis. Differing from the previous case, for the spin $3 / 2$ field we make

\footnotetext{
${ }^{3}$ In the linearized theory, the supervariation of (3) should vanish upon imposing other field equations.
} 
the special choice $\psi_{M}^{0}=D_{M} \epsilon_{0}$ (which is actually zero) and insert it into the RaritaSchwinger equations (3) (which are trivially satisfied). The same calculations which have been done to obtain (6) from (3), now implies that (3) becomes:

$$
\left(G_{M N}-T_{M N}\right) \Gamma^{N} \epsilon_{0}=0 .
$$

Note that to reach (6) from (3), we have only used the 4-form field equations which are also satisfied by the new background. Equations (6) are satisfied for every Majorana spinor $\epsilon$, because that background is chosen to obey Einstein equations. On the other hand (11) is correct only for the Killing spinor $\epsilon_{0}$, since then the RaritaSchwinger equations are trivially satisfied. When the indices of (11) refer to the orthonormal basis, in which by assumption only the diagonal entries of Einstein and energy momentum tensors are non-zero, it implies:

$$
G_{M N}-T_{M N}=0
$$

which are the Einstein equations (11) written in a slightly different form. Therefore we have proven that:

(i) if a chosen background solves the 4-form field equations (2),

(ii) if it is known that there exist a Killing spinor on this background,

(iii) if there exist an orthonormal basis in which the Einstein and energy momentum tensors have only diagonal entries;

then this background, which preserves some fraction of supersymmetries, also solves the Einstein equations of $\mathrm{D}=11$ supergravity.

The main advantage of the theorem is that, to find a solution of the second order Einstein equations, one can instead concentrate on the first order Killing spinor equations, which are of course easier to solve. It is not too difficult to write non-trivial ansatzs which solve the 4-form field equations from the beginning. Note that, for many of the known black p-brane solutions, assumed form of the geometry and other fields have the property (iii) (see for instance [5], [6]). This is because the metric of a static black hole solution depends on a single radial coordinate and usually has spherical symmetry . Therefore, it is not a big restriction to fulfill the conditions of

\footnotetext{
${ }^{4}$ The usual multi-source p-brane solutions do not obey the condition (iii), since the metrics depend on all transverse coordinates and the Ricci tensor has non-diagonal entries.
} 
the theorem.

Although explicitly proven for $\mathrm{D}=11$ supergravity, such a theorem can be generalized to any supergravity theory. To see this, let us remind that the expression

$$
\Gamma^{M N P} D_{N} D_{P} \epsilon=0
$$

where

$$
D_{M}=\nabla_{M}+\ldots
$$

is the supercovariant derivative of the supergravity at hand, $\epsilon$ is an arbitrary spinor and fields are evaluated on a bosonic background which obeys the equations of motion, is a generic condition for the linearized supersymmetry invariance. In (13), derivatives of the metric $g_{M N}$ appear only in the combination of Einstein tensor, due to the identity (8). Like in $\mathrm{D}=11$ case, the $\nabla_{M} \epsilon$ terms should cancel. Therefore, after imposing all but Einstein equations, (13) should become (6) (with the appropriate energy momentum tensor of the theory) which is also satisfied upon imposing Einstein equations; ensuring the linearized supersymmetry invariance.

With the information above, now we can generalize the theorem. Dropping the condition that the background satisfies Einstein equations but assuming the existence of a Killing spinor, one obtains, following (13), again (6) this time evaluated for the Killing spinor. With condition (iii), this implies the Einstein equations. Therefore, if we replace (i) with

$\left(i^{\prime}\right)$ if the background satisfies all but Einstein equations,

then together with conditions (ii) and (iii) above, the conclusions of the theorem should be valid for all supergravities.

Let us now illustrate how the theorem may be used for applications. Consider type IIB supergravity in 10-dimensions [7]. We focus on the metric $g_{M N}$ and anti-self dual 5-form field $F_{M N P Q R}$, and set all other fields to zero. The field equations which govern the dynamics may be written as:

$$
\begin{gathered}
R_{M N}=\frac{1}{96} F^{P Q R S}{ }_{M} F_{P Q R S N}, \\
d F=0, * F=-F
\end{gathered}
$$


where $*$ is the usual Hodge dual acting on differential forms. The Killing spinors, on a bosonic background, satisfy:

$$
\nabla_{M} \epsilon+\frac{i}{4 \times 480} \Gamma^{N P . . Q} \Gamma_{M} F_{N P . . Q} \epsilon=0,
$$

where $\epsilon$ is a chiral spinor $\Gamma_{11} \epsilon=\epsilon ; \Gamma_{11}=\Gamma_{\hat{0}} \ldots \Gamma_{\hat{g}}, \Gamma_{11}^{\dagger}=\Gamma_{11}$, and $\Gamma_{11}^{2}=I$. For illustrational purposes, it is sufficient to write a brane-like ansatz for the metric:

$$
d s^{2}=A(r)^{2}\left(-d t^{2}+d x_{1}^{2}+. .+d x_{3}^{2}\right)+B(r)^{2} d r^{2}+C(r)^{2} d \Omega_{5}^{2}(m),
$$

where $d \Omega_{5}^{2}(m)$ is the usual metric of $S_{5}(m)$; the 5 -sphere with inverse radius $m$. Note that the functions $A, B$ and $C$ depend only on the radial coordinate $r$. The Ricci tensor has only diagonal entries in the orthonormal basis $E^{\hat{i}}=A d x^{i}, E^{\hat{r}}=B d r$ and $E^{\hat{a}}=C e^{\hat{a}}$, where $x^{i}=t, x_{1}, . ., x_{3} ; e^{\hat{a}}$ is an orthonormal basis on $S_{5}(m)$. When we write the Killing spinor equations, all the spinors and hatted quantities will refer to the above orthonormal basis. We will also use the notation that, the indices $\mu, \nu, \sigma, \lambda$ refer to the coordinates $x^{i}, r$ and the indices $a, b, c, d, e$ refer to the coordinates of $S_{5}(m)$.

The 5-form field may initially be chosen to be a constant times the volume form on $S_{5}(m)$. The anti-self duality requires that, there should also be non-zero components along $x^{i}$ and $r$ directions. As a result one may write:

$$
\begin{gathered}
F_{\mu \nu \sigma \lambda \beta}=\frac{-q}{C^{5}} \epsilon_{\mu \nu \sigma \lambda \beta}, \\
F_{a b c d e}=\frac{q}{C^{5}} \epsilon_{a b c d e},
\end{gathered}
$$

where $q$ is a constant and the presence of function $C$ is due to the fact that the volume forms in (19) and (20) are determined by the metric (18). The field equations (16) for $F_{M N P Q R}$ are trivially satisfied. The background has now the properties $\left(i^{\prime}\right)$ and (iii) above. The parameter $q$ is related to the electric and magnetic charges of the background, which are identical due to the anti-self duality condition. On the other hand the Einstein equations (15) become:

$$
\begin{gathered}
-3\left(\frac{A^{\prime}}{A B}\right)^{2}-\frac{1}{A B}\left(\frac{A^{\prime}}{B}\right)^{\prime}-5 \frac{A^{\prime} C^{\prime}}{A C B^{2}}=-\frac{q^{2}}{4 C^{10}} \\
-\frac{4}{A B}\left(\frac{A^{\prime}}{B}\right)^{\prime}-\frac{5}{B C}\left(\frac{C^{\prime}}{B}\right)^{\prime}=-\frac{q^{2}}{4 C^{10}}
\end{gathered}
$$




$$
\frac{4 a^{2}}{C^{2}}-4\left(\frac{C^{\prime}}{B C}\right)^{2}-4 \frac{A^{\prime} C^{\prime}}{A C B^{2}}-\frac{1}{B C}\left(\frac{C^{\prime}}{B}\right)^{\prime}=\frac{q^{2}}{4 C^{10}}
$$

where' denotes the derivative with respect to $r$.

Let us now turn to the Killing spinor equations (17). We make $(5+5)$ splitting of the 10-dimensional gamma-matrices $\Gamma_{M}$ :

$$
\begin{aligned}
& \Gamma_{\mu}=\left(\gamma_{\mu} \times I \times \sigma_{1}\right), \\
& \Gamma_{a}=\left(I \times \Sigma_{a} \times \sigma_{2}\right),
\end{aligned}
$$

where $\gamma_{\mu}$ and $\Sigma_{a}$ are 5-dimensional gamma matrices and $\sigma_{1}, \sigma_{2}$ are the usual Pauli matrices. The chirality condition of the spinors becomes $\sigma_{3} \epsilon=\epsilon$. The Killing spinor equations may now be written explicitly as:

$$
\begin{gathered}
\partial_{i} \epsilon+\frac{A^{\prime}}{2 B} \gamma_{\hat{i}} \gamma_{\hat{r}} \epsilon+\frac{q A}{8 C^{5}} \gamma_{\hat{i}} \epsilon=0, \\
\partial_{r} \epsilon+\frac{q B}{8 C^{5}} \gamma_{\hat{r}} \epsilon=0, \\
\nabla_{a} \epsilon-\frac{i C^{\prime}}{2 B} e^{\hat{b}}{ }_{a} \Sigma_{\hat{b}} \gamma_{\hat{r}} \epsilon+\frac{i q}{8 C^{4}} e^{\hat{b}}{ }_{a} \Sigma_{\hat{b}} \epsilon=0,
\end{gathered}
$$

where $\nabla_{a}$ is the usual covariant derivative and $e^{\hat{b}}{ }_{a}$ is an orthonormal basis on $S_{5}(m)$. One can choose $\epsilon$ to be the Killing spinor on $S_{5}(m)$ satisfying:

$$
\nabla_{a} \epsilon+\frac{i m}{2} e^{\hat{b}} \Sigma_{\hat{b}} \epsilon=0
$$

Note that the indices on (28) and (29) refer to different spheres which are conformally related. Imposing

$$
\gamma_{\hat{r}} \epsilon=-\epsilon
$$

and

$$
\begin{gathered}
\frac{A^{\prime}}{B}=\frac{q A}{4 C^{5}}, \\
\frac{C^{\prime}}{B}=-\frac{q}{4 C^{4}}+m,
\end{gathered}
$$

the Killing spinor equations become integrable. Since the background has now Killing spinors and thus satisfies the conditions $\left(i^{\prime}\right),(i i)$ and $(i i i)$, the Einstein equations should also be trivially satisfied. Indeed, one can check that, when $A, B$ and $C$ obey (31) and (32), they also solve complicated Einstein equations (21)-(23). 
There are three independent functions and two differential equations which may be thought to imply that the functions are not constrained enough. However, the reparametrization invariance can be used to set $C=r$. Then, from (31) and (32), $A$ and $B$ can be solved as:

$$
\begin{aligned}
B & =\frac{4}{4 m-q r^{-4}} \\
A & =B^{-1 / 4}
\end{aligned}
$$

This is the self-dual black threebrane solution of Duff and Lu [8], written in a slightly different coordinates. To see this, one should make the coordinate change $4 m r^{4}-q \rightarrow r^{4}$ and introduce Euclidean coordinates for the transverse directions to the threebrane.

Note that, this way of solving field equations using Killing spinors is different from the methods which were used in the past in the context of Kaluza-Klein compactifications, see for instance [9]. It is very difficult to apply those methods to obtain a brane like solution. However, as we have illustrated, it is very natural to consider Killing spinor equations even to obtain brane solutions. Here, we have presented an example from IIB supergravity in 10-dimensions, but one can also obtain the M2 and M5 brane solutions of $\mathrm{D}=11$ supergravity.

We now use the theorem to find a new supersymmetric solution of $D=11$ supergravity. Basically, one may consider manifolds having Killing spinors (like anti-de Sitter (AdS) spaces or spheres) and write suitable ansatzs involving them. One of the simplest possible case that one may work is a space which has covariantly constant spinors like K3. K3 is a 4-dimensional Ricci flat Kähler manifold which has self dual (or anti-self dual) curvature tensor. It can be described by specifying 58 real constants and has no continuous isometries. The metric, which has self dual curvature, is not explicitly known but its existence is proven. Unlike a generic, oriented, 4-dimensional manifold, the holonomy group is just a copy of $S U(2)$ rather than $S O(4)=S U(2) \times S U(2)$. This can be seen by the decomposition of $S O(4)$ tensors with respect to their $S U(2)$ content. The curvature 2-form is an $S O(4)$ valued tensor, however, due to (anti) self duality, it has only non-zero components in one of the $S U(2)$ bundles. Therefore, the other copy of the $S U(2)$ bundle is flat. Because of this property, $K 3$ has two linearly independent covariantly constant $S U(2)$ spinors which are left or right handed according to the curvature tensor being self dual or anti-self dual. 
Now, consider the following background of $\mathrm{D}=11$ supergravity theory,

$$
\begin{gathered}
d s^{2}=A(r)^{2}\left(-d t^{2}+d x^{2}+d s_{K 3}^{2}\right)+B(r)^{2} d r^{2}+C(r)^{2} d \Omega_{4}^{2}(m), \\
F=3 q \epsilon_{4},
\end{gathered}
$$

where $d s_{K 3}^{2}$ is the line element on $K 3, \epsilon_{4}$ is the volume form on $S_{4}(m)$, the four sphere with inverse radius $m$, and $q$ is a real constant, which is proportional to the the magnetic charge of the background calculated as the integral of $F$ over $S_{4}(m)$. Note that, as before, the unknown functions $A, B$ and $C$ depend only on the radial coordinate $r$. We choose the following orthonormal basis on the tangent space, $E^{\hat{i}}=A d x^{i}$, $E^{\hat{\alpha}}=A e^{\hat{\alpha}}, E^{\hat{r}}=B d r$ and $E^{\hat{a}}=C e^{\hat{a}}$, where $x^{i}=(t, x), e^{\hat{\alpha}}$ is an orthonormal basis on $K 3$ and $e^{\hat{a}}$ is an orthonormal basis on $S_{4}(m)$. All the hatted indices and spinors will refer to this basis. We will also use the indices $\mu, \nu, \sigma \ldots$ to denote the coordinates $t, x, r$ and the coordinates of $K 3$. The indices $\alpha, \beta \ldots$ will refer to the coordinates of $K 3$ only.

It can easily be checked that the conditions (i) and (iii) of the theorem are satisfied by the background. Let us therefore consider the Killing spinor equations. We make the $(7+4)$ splitting of 11-dimensional gamma matrices $\Gamma_{A}$ as,

$$
\begin{gathered}
\Gamma_{\mu}=\left(\gamma_{\mu} \times \Sigma_{5}\right), \\
\Gamma_{a}=\left(I \times \Sigma_{a}\right),
\end{gathered}
$$

where $\gamma_{\mu}$ and $\Sigma_{a}$ are the 7 and 4-dimensional gamma matrices, respectively. The Killing spinor equations (10) can now be written explicitly as,

$$
\begin{gathered}
\partial_{i} \epsilon+\frac{A^{\prime}}{2 B} \gamma_{\hat{i}} \gamma_{\hat{r}} \epsilon+\frac{q A}{2 C^{4}} \gamma_{\hat{i}} \epsilon=0, \\
\nabla_{\alpha} \epsilon+\frac{A^{\prime}}{2 B} e^{\hat{\beta}}{ }_{\alpha} \gamma_{\hat{\beta}} \gamma_{\hat{r}} \epsilon+\frac{q A}{2 C^{4}} e^{\hat{\beta}_{\alpha}} \gamma_{\hat{\beta}} \epsilon=0, \\
\partial_{r} \epsilon+\frac{q B}{2 C^{4}} \gamma_{\hat{r}} \epsilon=0, \\
\nabla_{a} \epsilon+\frac{C^{\prime}}{2 B} e^{\hat{b}}{ }_{a} \Sigma_{\hat{b}} \Sigma_{5} \gamma_{\hat{r}} \epsilon-\frac{q}{C^{3}} e^{\hat{b}}{ }_{a} \Sigma_{\hat{b}} \Sigma_{5} \epsilon=0,
\end{gathered}
$$

where $\nabla_{\alpha}$ and $\nabla_{a}$ are covariant derivatives on $K 3$ and $S_{4}(m)$, respectively, and ' denotes differentiation with respect to $r$. One can choose $\epsilon$ to be the tensor product of covariantly constant spinors on $K 3$ and the Killing spinors on $S_{4}(m)$. Furthermore, it can also be chosen to be independent of the coordinates $t$ and $x$, and thus satisfies,

$$
\partial_{i} \epsilon=0,
$$




$$
\begin{gathered}
\nabla_{\alpha} \epsilon=0, \\
\nabla_{a} \epsilon-\frac{m}{2} e^{\hat{b}}{ }_{a} \Sigma_{\hat{b}} \Sigma_{5} \epsilon=0 .
\end{gathered}
$$

Note the difference between the Killing spinor equations (29) on $S_{5}$ and (45) on $S_{4}$. Equations (39)-(42) can be integrated if one also imposes,

$$
\gamma_{\hat{r}} \epsilon=-\epsilon,
$$

and

$$
\begin{gathered}
\frac{A^{\prime}}{B}=\frac{q A}{C^{4}}, \\
\frac{C^{\prime}}{B}=m-\frac{2 q}{C^{3}} .
\end{gathered}
$$

The projection imposed on $\epsilon$ is consistent with the equations (43), (44) and (45). Using the reparametrization invariance, one can set $C=r$. Solving for $A$ and $B$ we obtain the following supersymmetric solution,

$$
\begin{gathered}
d s^{2}=\left(m-\frac{2 q}{r^{3}}\right)^{1 / 3}\left(-d t^{2}+d x^{2}+d s_{K 3}^{2}\right)+\left(m-\frac{2 q}{r^{3}}\right)^{-2} d r^{2}+r^{2} d \Omega_{4}^{2}(m), \\
F=3 q \epsilon_{4},
\end{gathered}
$$

which represents a magnetically charged fivebrane wrapped on the manifold $K 3$. The horizon is located at $r=(2 q / m)^{1 / 3}$ and, unlike the horizon of the well known Güven's fivebrane [5], it seems to be a singular surface. One faces with the same kind of singularities when the fivebrane wraps a torus [10]. At spatial infinity, i.e. when $r \rightarrow \infty$, the solution approaches $R^{7} \times K 3$ and therefore related to the M-theory compactified on $K 3$. The mass can be calculated with respect to this background and turns out to be proportional to $q$. Due to supersymmetry, the mass and the charge saturate the Bogomolnyi bound and thus they are equal.

There are 8 Killing spinors, which can be constructed as the tensor product of the single Killing spinor of the space parametrized by $t, x, r$ obeying (46), 2 covariantly constant spinors of $K 3$ and 4 Killing spinors of $S_{4}(m)$. Therefore, the solution preserves $1 / 4$ 'th supersymmetries of the theory.

As a consequence of the supersymmetry, the new solution we obtained has also multi-source generalizations which can be found by defining a new radial parameter $R, R^{3}=m r^{3}-2 q$, and introducing cartesian coordinates, $\vec{R}$, on the Euclidean space 
transverse to the fivebranes. In this coordinate system, the fields of multi-source fivebranes having $K 3 \times R$ topology can be written as

$$
\begin{gathered}
d s^{2}=H^{-1 / 3}\left(-d t^{2}+d x^{2}+d s_{K 3}^{2}\right)+H^{2 / 3} d \vec{R} \cdot d \vec{R}, \\
F=* d H,
\end{gathered}
$$

where $*$ is the hodge dual in the transverse Euclidean space, $H$ is a generic harmonic function of the coordinates $\vec{R}$,

$$
H=1+\Sigma_{i} \frac{q_{i}}{\left|\vec{R}-\overrightarrow{R_{i}}\right|^{3}}
$$

and the constants $\vec{R}_{i}$ and $q_{i}$ denote the position and the magnetic charge of the $i$ 'th fivebrane, respectively.

This solution is very similar to the Güven's multi-source fivebrane, the only difference is that the 4 flat directions of the branes are replaced by $K 3$. This amounts to break 16 supersymmetries of the original solution by another half. Indeed, $K 3$ can be replaced by any other Ricci flat four dimensional manifold, however, a generic choice may break all the supersymmetries. The presence of $K 3$ also breaks the translational invariance along 4 directions on the branes (note that $K 3$ has no isometries). The effective theory describing the dynamics is a $d=2, N=8$ supersymmetric field theory. There are 24 fermionic and 12 bosonic zero modes which give 12 on-shell fermionic and bosonic degrees of freedom. The 9 of the bosonic zero modes are related to the broken translational invariance and 3 of them should come from the fluctuations of the four-form field on the fivebrane background.

Let us conclude by mentioning some other possible new solutions which may be obtained by using the Killing spinor equations. A simple modification is to replace the 5 -sphere $S_{5}$ in (18) by $S_{2} \times S_{3}, S_{1} \times S_{4}$ or by any other compact space which have Killing spinors and diagonal Ricci tensors. This may lead to threebrane solutions with different transverse spaces. Of course, this modification can be applied to all brane solutions. In some supergravities, one can also work with Calabi-Yau or other Kähler manifolds instead of spheres, which may give generalizations of compactifications studied in [1].

Another interesting choice is conformally AdS or de-Sitter type of geometries for the branes. When the conformal factors depend on a single radial coordinate 
transverse to the branes, these spaces will also have Killing spinors and diagonal Ricci tensors. This may give branes with AdS or de-Sitter topologies. One can also try to obtain time dependent cosmological type solutions, by changing the role played by the time coordinate $t$ and the radial coordinate $r$ in the above construction. These new possibilities will be studied elsewhere.

\section{Acknowledgements}

I would like to thank S. Deg̃er for reading the manuscript and helpful comments.

\section{References}

[1] M.J. Duff et.al. Phys. Rep. 259 (1995) 213,

[2] J.P. Gauntlett, Intersecting Branes, hep-th 9705011,

[3] E. Witten and D. Olive, Phys. Lett. 78B (1978) 97,

[4] E. Cremmer et.al. Phys. Lett. 76B (1978) 409,

[5] R. Güven, Phys. Lett. 276B (1992) 49,

[6] G. Horowitz and A. Strominger, Nucl. Phys. B 360 (1991) 197,

[7] J.H. Schwarz, Nucl. Phys. 226B (1983) 269,

[8] M.J. Duff and J.X. Lu, Phys. Lett. 273B (1991) 409,

[9] M.J. Duff et.al. Phys. Rep. 130 (1986) 1,

[10] G. W. Gibbons, Wrapping Branes in Space and Time,hep-th 9803206,

[11] P. Candelas et.al. Nucl. Phys. 258B (1985) 46. 